\title{
Prácticas, percepciones y emociones de docentes de Argentina en tiempos de pandemia Covid-19
}

\section{Practices, perceptions and emotions of teachers in Argentina in times of the Covid-19 pandemic}

Gabriela Vergara. CONICET-UNVM, UNRaf, Argentina. gabivergaramattar@gmail.com

Vanina Fraire.

UTN-San Francisco, (Córdoba, Argentina). vafraire@gmail.com

Agustina Manavella. CONICET_UNVM, Argentina. agumanavella@gmail.com

Solana Salessi.

CIT-CONICET-UNRaf, UNRaf, Argentina. solanasalessi@gmail.com

\section{RESUMEN.}

El artículo presenta un análisis de prácticas, percepciones y emociones en torno a las correcciones de docentes de Córdoba, Buenos Aires y Santa Fe (Argentina), que durante el Aislamiento Social Preventivo y Obligatorio (ASPO) de 2020, realizaron una enseñanza a distancia por emergencia sanitaria. Desde una sociología de los cuerpos y las emociones, se describen los resultados de 387 casos, que respondieron una encuesta on line durante junio de 2020, diferenciando por niveles educativos y tipos de gestión. El nivel secundario o medio muestra mayor carga de trabajo, con percepciones favorables sobre lo realizado, aunque con bajos niveles de innovación. El lugar de lo afectivo en las correcciones se destaca en el nivel inicial. Los y las docentes se reconocen frente a un trabajo que cambia cuantitativa y cualitativamente, pero frente al cual cumplen y creen poder adaptarse a los cambios tecnológicos, sintiendo principalmente alegría y orgullo por lo que hacen. Se sugiere avanzar en el estudio de la construcción de nuevas habilidades y la relación entre cantidad y cualidad de instancias evaluativas en educación secundaria.

\section{PALABRAS CLAVE.}

Prácticas, percepciones, emociones, docentes, COVID-19.

\section{ABSTRACT.}

The article presents an analysis of practices, perceptions and emotions around the corrections of teachers in Cordoba, Buenos Aires and Santa Fe (Argentina), who during the Preventive and Compulsory Social Isolation (ASPO) during 2020, carried out distance teaching due to health emergency. From a sociology of bodies and emotions, we describe the results of 387

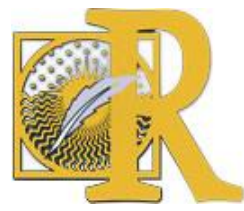


cases, who responded to an online survey during June 2020, differentiating by educational levels and types of management. The secondary or middle level shows a greater workload, with favorable perceptions of what has been done, although with low levels of innovation. The place of affective aspects in the corrections is highlighted at the initial level. Teachers recognize that they are faced with a job that changes quantitatively and qualitatively, but that they comply with and believe they can adapt to technological changes, feeling mainly joy and pride in what they do. It is suggested to deepen the place of new skills in secondary school and the relationship between quantity and quality in the tests.

\section{KEY WORDS.}

Practices, perceptions, emotions, teachers, COVID-19.

\section{Introducción.}

El mundo del trabajo en tiempos de pandemia por Covid-19 mostró reconfiguraciones en las formas de concretarlo, consolidando y extendiendo el "home-office" o el "teletrabajo" en actividades en que fue posible su implementación. Tal el caso de la docencia, dado que para evitar la propagación del virus, se aplicó en la mayoría de los países latinoamericanos una modalidad de educación a distancia por emergencia sanitaria (Baptista Lucio et al., 2020) recurriendo a la mediación tecnológica. En el pasaje a la virtualidad, los y las docentes debieron modificar sus prácticas educativas cotidianas: incorporar la implementación de herramientas, materiales y recursos digitales, construir competencias y habilidades en el manejo y uso de tecnologías de la información y comunicación (TIC), adaptar el currículum, priorizar la enseñanza y la evaluación de ciertos contenidos por sobre otros, como así también la manera de abordar las clases, las interacciones e instancias evaluativas (SITEAL, 2020). En Argentina ${ }^{1}$, a partir de la resolución $N^{\circ} 108 / 2020$, del 16 de marzo de 2020 del Ministerio de Educación de la Nación, en el marco del Aislamiento Social Preventivo y Obligatorio (ASPO) se estableció la "suspensión temporal del dictado de clases presenciales en los niveles inicial, primario, secundario en todas sus modalidades e institutos de educación superior, por catorce días corridos a partir del 16 de marzo" (Artículo 1, Ministerio de Educación, Res. N¹08/2020). Esta medida se extendió durante todo 2020, estableciéndose modificaciones en las calificaciones. Puntualmente, el Consejo Federal de Educación de Argentina (en adelante, CFE), en la Resolución N³63/20 del 19 de mayo de 2020 dispuso variados recursos pedagógicos, en vistas a una evaluación formativa, amplia e integral, orientada a mejorar los procesos de enseñanza y aprendizaje, promover procesos autoevaluativos en los y las estudiantes y, a interpretar la singularidad de los procesos de enseñanza y acompañamiento desplegados durante este período. Se resolvió que en contexto de pandemia no iba a haber calificaciones numéricas, por lo tanto, las evaluaciones debían centrarse en el acompañamiento, seguimiento, registro y devolución a familias y estudiantes de los procesos de aprendizaje (Anexo I, Res. №108/20; SITEAL, 2020). El concepto de evaluación formativa se vincula al de evaluación continua, que recopila sistemáticamente información del proceso de aprendizaje de cada estudiante. Esto asegura el desarrollo de mecanismos de autorregulación para alcanzar nuevas metas de aprendizaje (Pérez Pino et al., 2017).

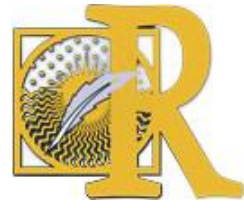

Fecha de recepción: 28-04-2021 Fecha de aceptación: 30-04-2021

Vergara, G., Fraire, V., Manavella, A. \& Salessi, S. Prácticas, percepciones y emociones de docentes de Argentina en tiempos de pandemia Covid-19

International Journal of Educational Research and Innovation (IJERI), 15, 568-584 ISSN: 2386-4303 DOI https://doi.org/10.46661/ijeri.5903 
Diversas investigaciones abordaron la situación del trabajo docente y las emociones. Algunos estudios relevaron niveles medios de estrés percibido, con valores más elevados en mujeres que varones (Oducado et al., 2021), sintiendo tensión y agotamiento emocional, sobrecarga de tareas y demanda externa de trabajo, temor y preocupación por el futuro, presión por los plazos de tiempo establecidos por la institución educativa y por las responsabilidades a cumplir (Gómez Dávalos y Rodríguez Fernández, 2020).

En relación con la labor docente, las tareas más realizadas fueron la revisión y evaluación del desarrollo de contenidos. En cuanto a la comunicación entre docentes y estudiantes, se destacó la ausencia de herramientas sincrónicas para la comunicación directa y en tiempo real. El estado emocional que experimentaron al iniciar las clases no presenciales, fue de confianza y a la vez estrés, ansiedad y miedo de asumir su tarea en la virtualidad (Picón et al., 2020).

En México, se realizó en abril de 2020 de manera on line, la Encuesta Nacional de Docentes ante el Covid-19 (END-Covid-19) a 2253 docentes de educación básica (preescolar, primaria, secundaria y bachillerato) del Sistema Educativo Nacional. El estudio identificó el uso de herramientas digitales (WhatsApp, Facebook) y recursos tradicionales (fotocopias y libros de texto), encontrando diferencias por región geográfica y nivel educativo por sobre la edad o el género. También se midieron sentimientos positivos acerca de la posibilidad de recibir acompañamiento en ambientes digitales, siendo el nivel primario el que reportó mayor indecisión a la hora de capacitarse en herramientas digitales y plataformas. En cuanto a las estrategias didácticas, el $65 \%$ manifestó sentimientos positivos o, satisfacción por lo que realizaban, aunque se detectó que, a mayor edad, se incrementan los sentimientos negativos (Baptista Lucio et al., 2020).

En Argentina Narodowski, Volman y Braga (2020), investigaron la situación de las familias y los alumnos durante el aislamiento. El universo de análisis estuvo conformado por 262 padres o tutores de distintas escuelas del país de nivel primario, pertenecientes al ámbito urbano, de gestión estatal y educación común. Entre los resultados de la investigación, se destaca que 6 de cada 10 alumnos $(61,4 \%)$ recibieron siempre correcciones o devoluciones de un docente. Las escuelas de jornada extendida/completa presentaron, en promedio, 10 puntos porcentuales más en la categoría de corrección docente "siempre" en comparación con las escuelas de jornada simple. A pesar de esta diferencia, se mantuvo muy similar el porcentaje de alumnos que no recibió nunca una corrección o devolución docente. En el momento de la recogida de datos, solo en el $11,5 \%$ de los casos los alumnos fueron evaluados mediante pruebas o exámenes durante el período transcurrido desde el inicio de la pandemia. En tres de cada cuatro casos se optó por una calificación conceptual (y no numérica), lo que indicaría una preferencia pedagógica por las evaluaciones de proceso y no de resultados. Entre las consideraciones finales, los autores destacaron que el esfuerzo por parte de docentes de brindar devoluciones y correcciones a los estudiantes aumentó en escuelas de jornada completa, lo que explica que, a menor carga laboral docente, mayor compromiso con la devolución de tareas.

En este artículo se describen prácticas, percepciones y emociones vinculadas con la corrección/evaluación de actividades de estudiantes. Los datos surgen de la "Encuesta acerca de la enseñanza en tiempos de cuarentena para docentes de Educación Inicial,

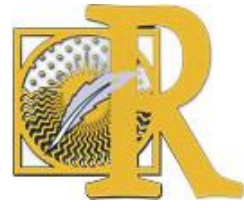

Fecha de recepción: 28-04-2021 Fecha de aceptación: 30-04-2021

Vergara, G., Fraire, V., Manavella, A. \& Salessi, S. Prácticas, percepciones y emociones de docentes de Argentina en tiempos de pandemia Covid-19 
Primaria y Secundaria (Argentina, 2020)" aplicada de manera on line durante el mes de junio de $2020^{2}$. La pregunta que orientó el presente análisis fue acerca de la relación entre las percepciones y emociones que genera corregir en tiempos de ASPO.

\section{2- Marco teórico de la investigación.}

La indagación por la labor docente en tiempos de pandemia se inscribe en un interrogante más general acerca del mundo del trabajo, los procesos de estructuración social y las sensibilidades de agentes sociales.

Cuerpos y emociones en la Sociología y Antropología han sido abordados desde los clásicos, profundizándose su abordaje en las últimas décadas del siglo pasado, con diversos énfasis y orientaciones (Turner, 1994; Bericat Alastuey, 2000; Le Breton, 2002). Una posible clasificación de los estudios sobre las emociones diferencian entre a)naturalistas, -para quienes la cultura sólo modela la intensidad y expresividad emotivas siendo su origen de carácter biológico-; b)constructivistas no radicales -que asumen la dualidad de las emociones en tanto resultantes de procesos neurofisiológicos como sociohistóricos-; y c)constructivistas radicales, quienes sostienen que las emociones se forman en las interacciones sociales, permitiendo la aprehensión de patrones socio-comunicacionales vinculados con la expresividad de las mismas a partir de ciertos factores de enclasamiento, tales como la clase, el género, la edad, la etnia (Luna Zamora, 2010).

Una mirada sociológica de los cuerpos y las emociones (Scribano, 2012), implica asumir que el cuerpo es una trama desde donde el mundo se aprehende, se siente y en el que se actúa, en el marco de procesos de estructuración social capitalistas que reconfiguran sensibilidades (Scribano, 2010). Es decir, "un ser corpóreo, dotado de fuerzas naturales, vital, real, sensorial, objetivo significa que tiene objetos reales, sensoriales como objeto de su ser, de su expresión vital o que solo puede expresar su vida en objetos reales, sensoriales" (Marx, 2004: 198). La corporeidad y la sensorialidad presentan conexiones recíprocas que se hacen prácticas sociales espacio/temporalmente y estructuralmente situadas, siendo las emociones resultantes de las relaciones entre impresiones, percepciones y sensaciones (Scribano, 2007). Esa condición corporal se sostiene en la interrelación de tres dimensiones: a) una de carácter orgánico/biológico -con los procesos y funciones vitales, desde el nacimiento, el crecimiento, la reproducción y la muerte, los cuales también se construyen socialmente-; b) otra de tipo subjetivo (el self o el 'yo corporeizado') y, c) los aprendizajes sociales cognitivoafectivos, las incorporaciones y apropiaciones de hábitos, prácticas, gestos, lenguajes, códigos. Estas facetas forman tramas corporales (Vergara, 2011) combinadas con la biografía, por un lado, como condensación y síntesis de las vivencias y experiencias que articulan a su vez, lo particular e individual de cada agente con los procesos socio-históricos en los que ha vivido; es el cuerpo en el tiempo. Por otro, el espacio, como posiciones, condiciones y disposiciones de acción, desde donde también se constituye la forma de conocer y sentir el mundo, combinando aspectos neurofisiológicos y otros derivados de construcciones sociales, que se vuelven prácticas, acciones. En otros lugares, hemos analizado cómo el miedo se configura según las experiencias de clase (Vergara, 2013) y el orgullo puede ser el anverso de la resignación cuando se trata del lugar o barrio en que se vive (Fraire, 2015).

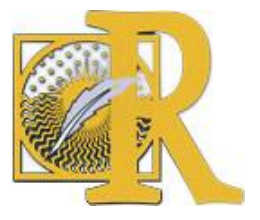

Fecha de recepción: 28-04-2021 Fecha de aceptación: 30-04-2021 Vergara, G., Fraire, V., Manavella, A. \& Salessi, S. Prácticas, percepciones y emociones de docentes de Argentina en tiempos de pandemia Covid-19 International Journal of Educational Research and Innovation (IJERI), 15, 568-584 ISSN: 2386-4303 DOI https://doi.org/10.46661/ijeri.5903 
A partir de la relación cuerpo-mundo, las percepciones se conectan con impresiones y sensaciones, que se organizan según patrones socioestructurales de interpretación y tipificación generados desde los lugares sociales -diferentes y diferenciables- que ocupan los cuerpos (Vergara, 2015). Por esto, la percepción no es la de alguien aislado, externo y puramente racional, como un observador que tiene frente a sí, una cosa (Bourdieu, 1999; 2003). Desde esta mirada teórica general, nos aproximamos a las metamorfosis del mundo del trabajo (Antunes, 2005) en general y al trabajo docente en particular, en tiempos de pandemia, asumiendo que la modalidad de educación a distancia por emergencia sanitaria, implicó profundos cambios en las prácticas espacio/temporales situadas. Dichos vectores inciden en las formas de percibir y sentir la vida cotidiana, la labor de enseñar.

\section{Material y métodos.}

\subsection{Diseño.}

Se realizó un estudio exploratorio de alcance descriptivo enmarcado en los lineamientos de la estrategia mixta.

\subsection{Participantes.}

La muestra no probabilística de 387 casos quedó conformada por un $87 \%$ de mujeres. La media de edad se ubicó en 40.94 ( $D E=8.67)$. El 44\% tenía hasta 9 años de antigüedad; el $30 \%$ entre $10-19$ años y el $19 \%$ entre $20-29$ años. El $68 \%$ convivía con otra persona (en pareja o matrimonio), mientras que el $22 \%$ se encontraba soltera/o. En el grupo etario de 1929 años, la mitad se declaró soltero/a, mientras que en el grupo de 30-39, el 70\% estaba casado/a; en el de $40-49$ el $75 \%$ estaba casado/a y en 50 y más, el $62 \%$ estaba casado/a, aumentando en este rango etario la presencia de separados/as/divorciados/as al $23 \%$. El $41 \%$ no tenía hijos/as; mientras que el $51 \%$ tenía 1-2 hijos/as. El rango etario de 19-29 mostró el mayor valor de sin hijos (74\%), mientras que entre 40-49 se registró el mayor valor de 2 hijos (38\%). En el $68 \%$ de los casos había 2 proveedores en el hogar. Entre los $30-50$ años el porcentaje de 2 proveedores era del $73 \%$. El $25 \%$ declaró que en el hogar había sólo una persona aportante, siendo el rango de 50 años y más donde se registró el principal valor, $36 \%$ (en coincidencia con el grupo etario que más porcentaje de personas separadas/divorciadas registra). Casi la mitad se percibió como jefe o jefa del hogar en función de los ingresos, pero el 42\% manifestó que era el cónyuge o pareja. Dentro del grupo etario de 30-50, casi la mitad afirmó que la jefatura del hogar por ingresos era del cónyuge o pareja (es decir, hogares nucleares biparentales, con 2 proveedores y con 2 hijos principalmente). El 68\% de los casos se desempeñaba en un establecimiento de gestión pública y, un $25 \%$ en gestión privada con aporte estatal. El $57 \%$ trabajaba en el nivel secundario, el $33 \%$ en primario y el $10 \%$ en nivel inicial.

\subsection{Instrumento.}

Tomando como base los conceptos de prácticas, percepciones y emociones, se diseñó un cuestionario cerrado, el cual quedó conformado por seis módulos. El primero abarcó información sociodemográfica (sexo, edad, estado civil, cargo docente, antigüedad, nivel en que se desempeña, tipo de gestión del establecimiento, entre otros). A partir de las

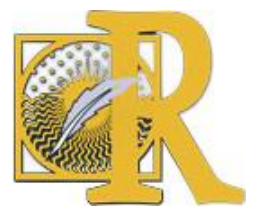


distinciones conceptuales se indagó sobre prácticas tales como desarrollo de contenidos, actividades para resolver, correcciones, evaluaciones y comunicación con padres de estudiantes, con el equipo directivo y con pares docentes. En cuanto a las percepciones se preguntó acerca de la cantidad y los criterios de selección de los contenidos, el formato o tipo de actividades propuestos a estudiantes, los criterios de corrección y de devoluciones a estudiantes, de selección de contenidos a evaluar y las emociones experimentadas durante las correcciones e instancias de evaluación. Junto a esto se elaboraron un conjunto de afirmaciones tendientes a evaluar niveles de acuerdo en torno al uso de la tecnología en la docencia, las posibilidades de corrección, la relación con pares, entre otros. En el último módulo de la encuesta se abordó el uso de herramientas, programas y aplicaciones antes de la cuarentena, tales como videollamada, redes sociales, home banking, entre otras. También se recolectó información sobre dispositivos tecnológicos adquiridos e implementados en la labor docente durante la cuarentena.

\subsection{Estrategia de análisis de los datos.}

La información recolectada se analizó en función de la edad, la antigüedad laboral, el nivel educativo y el tipo de gestión del establecimiento (público o privado). Se consideró relevante la distinción por nivel educativo en relación con la evaluación y la corrección, dadas las características propias de cada etapa formativa. El nivel educativo permite tomar en cuenta la influencia del entorno docente, las características de la enseñanza en cada ciclo, condicionando e influyendo en las prácticas, percepciones y emociones, acotadas a la corrección, en este caso. En cuanto al tipo de gestión, interesó identificar si había diferencias atento a criterios de calidad, seguimiento de estudiantes, entre otros factores. Considerando el nivel de medición utilizado para las variables de interés (nominal u ordinal) se computaron tablas de contingencia, pruebas de asociación con el estadístico chi cuadrado de Pearson, pruebas de diferencia de medias con el estadístico t de Student y ANOVA de un factor, según corresponda. Para la ejecución de los análisis se utilizó el software SPSS versión 23.

\section{Resultados.}

A continuación, se presentan los resultados organizados en 4 dimensiones: a) variables de contexto general; b) características de las prácticas de corrección; c) percepciones y, d) emociones de la corrección.

La cantidad total de horas semanales que casi la mitad (46.3\%) de los y las respondientes le dedicó al trabajo docente en tiempos de cuarentena (tales como preparación de clases, tiempo de clase por videollamada, para corregir, para consultas, etc.), fue de más de 32 horas semanales. En el nivel inicial el mayor porcentaje de respuestas fue en el rango de 13-24 horas, seguido por más de 32, no habiendo diferencia entre tipo de gestión. En el primario, el rango horario que casi 4 de 10 docentes eligió, fue el de mayor carga (más de 32 horas), seguido por privado, en el rango de 25-32. Esta tendencia se mantuvo en el secundario, con leve diferencia entre tipos de gestión. En cuanto a la distribución horaria del trabajo, más del $80 \%$ consideró que fue completamente diferente $(53,7 \%)$ o diferente $(34 \%)$ comparado con su trabajo antes de la cuarentena.

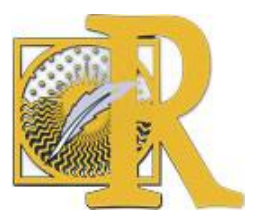

Fecha de recepción: 28-04-2021 Fecha de aceptación: 30-04-2021 


\section{Las prácticas de corrección por nivel educativo.}

En el $64 \%$ de los casos, los y las docentes indicaron que corregían más que antes de la cuarentena. En la Tabla 1., se presentan los resultados desde tres aspectos que relevó la encuesta, a saber: (a) cantidad de corrección durante la "cuarentena", comparada con el periodo anterior; (b) el nivel de importancia de los contenidos a evaluar o nuevas habilidades y (c) el nivel de contenidos o aspectos emocionales, considerando nivel educativo y tipo de gestión:

Tabla 1. Prácticas de corrección según nivel educativo y tipo de gestión.

\begin{tabular}{|c|c|c|c|c|c|c|c|}
\hline \multirow{2}{*}{ Variables } & \multirow{2}{*}{$\begin{array}{l}\text { Nivel educativo } \\
\text { Tipo de gestión }\end{array}$} & \multicolumn{2}{|l|}{ Inicial } & \multicolumn{2}{|l|}{ Primario } & \multicolumn{2}{|c|}{ Secundario } \\
\hline & & PÚB. \% & PRIV. \% & PÚB. \% & PRIV. \% & PÚB. \% & PRIV.\% \\
\hline \multirow{4}{*}{$\begin{array}{l}\text { Cantidad de } \\
\text { correcciones } \\
\text { durante la } \\
\text { cuarentena }\end{array}$} & Igual que antes & 20,7 & 10 & 28,6 & 22,2 & 19,3 & 15,5 \\
\hline & Más que antes & 58,6 & $40 \%$ & 54,1 & 59,3 & 68,1 & 76,2 \\
\hline & Menos que antes & 0 & 0 & 14,3 & 14,8 & 12 & 7,1 \\
\hline & $\begin{array}{lr}\text { No hay en } & \text { estas } \\
\text { instancias } & \text { de } \\
\text { enseñanza } & \end{array}$ & 20,7 & 50 & 3,1 & 3,7 & 0,70 & 1,2 \\
\hline \multirow{4}{*}{$\begin{array}{l}\text { Correcciones } \\
\text { según } \\
\text { contenidos y/o } \\
\text { emoción }\end{array}$} & Sólo contenido & 6,90 & 0 & 21,40 & 33,30 & 37,80 & 41,70 \\
\hline & $\begin{array}{l}\text { Sólo emoción/ } \\
\text { motivación }\end{array}$ & 62,10 & 70,0 & 29,60 & 22,20 & 16,30 & 17,90 \\
\hline & $\begin{array}{l}\text { Contenido } \quad \text { y } \\
\text { emoción/ } \\
\text { motivación }\end{array}$ & 13,80 & 0 & 43,90 & 40,70 & 42,20 & 39,30 \\
\hline & No corrige & 17,20 & 30,00 & 5,10 & 3,70 & 3,70 & 1,20 \\
\hline \multirow{6}{*}{$\begin{array}{l}\text { Correcciones } \\
\text { según } \\
\text { importancia de } \\
\text { contenidos u } \\
\text { otras } \\
\text { habilidades }\end{array}$} & Todos & 3,40 & 0 & 3,10 & 11,1 & 3,7 & 3,6 \\
\hline & $\begin{array}{l}\text { Sólo los más } \\
\text { importantes }\end{array}$ & 27,6 & 10 & 24,5 & 29,6 & 23 & 20 \\
\hline & $\begin{array}{l}\text { Algunos, aunque no } \\
\text { sean importantes }\end{array}$ & 0 & 10,00 & 3,10 & 0,00 & 1,50 & 2,40 \\
\hline & $\begin{array}{l}\text { Evalúo otras } \\
\text { /nuevas habilidades }\end{array}$ & 27,60 & 10,00 & 29,60 & 14,80 & 30,40 & 28,6 \\
\hline & No evalúo & 27,60 & 70,00 & 21,40 & 14,80 & 14,80 & 20,2 \\
\hline & $\begin{array}{l}\text { Más importantes y } \\
\text { otras habilidades }\end{array}$ & 13,80 & 0,00 & 18,40 & 29,60 & 26,70 & 25 \\
\hline
\end{tabular}

Fuente: elaboración propia. 


\section{INTERNATIONAL JOURNAL OF EDUCATIONAL \\ RESEARCH AND INNOVATION \\ REVISTA INTERNACIONAL DE INVESTIGACIÓN

En cuanto a la cantidad de correcciones realizadas, se encontró una asociación estadísticamente significativa con el nivel educativo $\left(x^{2}=74,76 ; p<0.00\right)$, aunque no con el tipo de gestión del establecimiento $\left(x^{2}=3.37 ; p>0.05\right)$.

La inspección de la Tabla 1 muestra que nivel secundario de gestión privado es el que presentó el mayor porcentaje (76.2\%), seguido por secundario público y primario privado. La corrección considerando aspectos emocionales/motivacionales tuvo en el nivel inicial su nivel más elevado (70\%), junto con la no corrección (30\%). En el primario se destacó una opción combinada de aspectos de contenido y emocionales/motivacionales (44\%), registrándose porcentajes levemente inferiores en el secundario. En cuanto al tercer aspecto, aproximadamente 1 de cada 4 docentes en los 3 niveles centró las instancias de evaluación en aspectos más importantes, mientras que, otro porcentaje similar se centró en otras 0 nuevas habilidades, habiendo una leve diferencia al aumentar los niveles educativos. Docentes de nivel primario privado $(29,6 \%)$, secundario público $(26,7 \%)$ y secundario privado $(25 \%)$ combinaron dos opciones de respuesta (aspectos importantes y nuevas habilidades). En cuanto al tipo de gestión del establecimiento no se observan diferencias significativas.

\subsection{Las percepciones sobre las correcciones.}

Las percepciones en tanto esquemas de clasificación y organización de las impresiones sensoriales fueron consideradas, por un lado, en relación con el contexto de pandemia y lo tecnológico y; por otro, con la práctica de la corrección en sí misma, tal como se presentan en la Tabla 2:

Tabla 2. Percepciones sobre la corrección.

\begin{tabular}{|c|c|c|c|c|c|c|c|}
\hline \multirow{2}{*}{ Percepciones } & Nivel educativo & \multicolumn{2}{|c|}{ INICIAL } & \multicolumn{2}{|c|}{ PRIMARIO } & \multicolumn{2}{c|}{ SECUNDARIO } \\
\cline { 2 - 7 } & Categorías & PÚB. \% & PRIV.\% & PÚB.\% & PRIV.\% & PÚB.\% & PRIV.\% \\
\hline \multirow{2}{*}{$\begin{array}{c}\text { "No puedo } \\
\text { corregir las } \\
\text { actividades en la } \\
\text { computadora" }\end{array}$} & $\begin{array}{c}\text { Acuerdo } \\
\text { desacuerdo }\end{array}$ & $13,8 \%$ & $\mathbf{4 0} \%$ & $19,4 \%$ & $3,7 \%$ & $18,5 \%$ & $13,1 \%$ \\
\cline { 2 - 8 } & Desacuerdo & $62,1 \%$ & $10 \%$ & $\mathbf{5 0} \%$ & $\mathbf{8 5 , 2} \%$ & $\mathbf{6 0 \%}$ & $\mathbf{6 9} \%$ \\
\hline \multirow{2}{*}{$\begin{array}{c}\text { "Puedo evaluar } \\
\text { a mis } \\
\text { estudiantes en } \\
\text { estas } \\
\text { circunstancias" }\end{array}$} & $\begin{array}{c}\text { Ni acuerdo ni } \\
\text { desacuerdo }\end{array}$ & $24,1 \%$ & $20 \%$ & $27,6 \%$ & $44,4 \%$ & $34,8 \%$ & $23,8 \%$ \\
\cline { 2 - 8 } & Desacuerdo & $37,9 \%$ & $\mathbf{7 0} \%$ & $\mathbf{4 0 , 8} \%$ & $25,9 \%$ & $33,3 \%$ & $\mathbf{4 6 , 4} \%$ \\
\hline
\end{tabular}




\begin{tabular}{|c|c|c|c|c|c|c|c|}
\hline \multirow{2}{*}{ Percepciones } & \multirow{2}{*}{$\frac{\text { Nivel educativo }}{\text { Categorías }}$} & \multicolumn{2}{|c|}{ INICIAL } & \multicolumn{2}{|c|}{ PRIMARIO } & \multicolumn{2}{|c|}{ SECUNDARIO } \\
\hline & & PÚB. \% & PRIV.\% & PÚB.\% & PRIV.\% & PÚB.\% & PRIV.\% \\
\hline \multirow{4}{*}{$\begin{array}{l}\text { Nivel de } \\
\text { adecuación de } \\
\text { correcciones }\end{array}$} & Adecuadas & 51,7 & 30 & 77,6 & 81,5 & 79,3 & 86,9 \\
\hline & Inadecuadas & 3,4 & 0 & 2 & 0 & 3,7 & 2,4 \\
\hline & $\begin{array}{l}\mathrm{Ni} \text { adecuadas / ni } \\
\text { inadecuadas }\end{array}$ & 20,7 & 30 & 9,2 & 7,4 & 14,8 & 10,7 \\
\hline & No aplica a mi caso & 24,1 & 40 & 11,2 & 11,1 & 2,2 & 0 \\
\hline \multirow{4}{*}{$\begin{array}{l}\text { Grado de } \\
\text { conformidad con } \\
\text { el propio trabajo }\end{array}$} & $\begin{array}{l}\text { Hago apenas, lo que } \\
\text { puedo hacer }\end{array}$ & 13,8 & 30 & 15,3 & 0 & 12,6 & 14,3 \\
\hline & $\begin{array}{l}\text { Cumplo con lo que } \\
\text { debo hacer como } \\
\text { docente }\end{array}$ & 41,4 & 20 & 55,1 & 70,4 & 59,3 & 59,5 \\
\hline & Hago innovaciones & 17,2 & 10 & 22,4 & 25,9 & 23 & 22,6 \\
\hline & No aplica a mi caso & 27,6 & 40 & 7,1 & 3,7 & 5,2 & 3,6 \\
\hline
\end{tabular}

Fuente: elaboración propia.

En relación con la frase "no puedo corregir en la computadora", se buscó identificar esquemas de clasificación en torno a una práctica docente concreta. La prueba ANOVA de un factor no arrojó diferencias estadísticamente significativas por nivel educativo $\left(F_{(387 ; 2)}=1.08 ; p>0.05\right)$. No obstante, sí se registraron diferencias en torno al tipo de gestión $(\mathrm{t}(387 ; 256)=-2.25, \mathrm{p}=0.05)$, siendo levemente superior en privado.

Un análisis más minucioso de los datos muestra que el $60 \%$ se ubicó en desacuerdo con la frase, el $23 \%$ no acordó ni estuvo en desacuerdo y el $17 \%$ estuvo de acuerdo con la frase. Considerando niveles y tipo de gestión se destacan los porcentajes de desacuerdo a "no poder" en primario $(85.2 \%)$ y secundario $(69 \%)$ privados, mientras que el mayor porcentaje de acuerdo se dio en el inicial privado.

La otra frase indagó por las posibilidades de evaluar en las circunstancias de educación a distancia en contexto de pandemia. El 39\% expresó desacuerdo, seguido por el $31 \%$ que acordó con la frase. En primer lugar, se ubicó el privado inicial (70\%), seguido por secundario privado $(46,4 \%)$ y primario público $(40,8 \%)$. No se encontraron diferencias estadísticamente significativas ni por tipo de gestión $\left(\mathrm{t}_{(387 ; 237)}=-0.91, \mathrm{p}>0.05\right)$, ni por nivel educativo $\left(\mathrm{F}_{(387 ; 2)=}\right.$ $0.213 ; p>0.05)$.

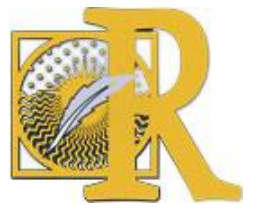


En cuanto a las percepciones sobre el nivel de adecuación de las correcciones que realizaban, el 77\% las identificó como "adecuadas", el 13\% "ni adecuadas/ni inadecuadas", el $7 \%$ expresó que no aplica a su caso y sólo el 3\% inadecuadas. Según el tipo de gestión se registró mayor porcentaje de adecuación de las correcciones en primario (81,5\%) y secundario privados $(86,9 \%)$. El análisis de la prueba Chi Cuadrado de Pearson revela que hay una asociación estadísticamente significativa con el nivel educativo $\left(X^{2}=74.76 ; p>0.0\right)$; no así con el tipo de gestión $\left(x^{2}=3.37 ; p>0.05\right)$.

En cuanto al último aspecto, sobre la conformidad con el propio trabajo, el 56\% identificó cumplir con su labor docente, el $22 \%$ hizo innovaciones y se consideró una persona creativa, en tanto que el $14 \%$ hacía lo que podía. Considerando los niveles y tipos de gestión no se encontraron asociaciones estadísticamente significativas $\left(X^{2}=3.80 ; p>0.05 ;\left(X^{2}=4\right.\right.$. 72; $p$ $>0.05$, respectivamente). Sin embargo, el análisis de frecuencia indica que el primario privado $(70,4 \%)$ obtuvo el mayor porcentaje.

4.3. Las emociones al corregir según nivel educativo

En la encuesta se podían marcar hasta tres respuestas, pero casi la mitad de los y las respondientes sólo seleccionaron una. De esta primera aproximación se destacaron "alegría" $(21,4 \%)$, "impotencia" $(14,7 \%)$ y "orgullo" $(11 \%)$.

Teniendo en cuenta las emociones por cercanía en su orientación, alegría y orgullo dieron cuenta del estado emocional en torno a las correcciones en 4 de cada 10 docentes $(41 \%$; $32 \%$ por separado y $9 \%$ en respuestas combinadas). Además, se registró un $12 \%$ de estados emocionales ambiguos (cuando la respuesta combinó impotencia y orgullo o alegría y miedo, por ejemplo.) En tanto que emociones como impotencia, miedo, enojo, vergüenza, tristeza, reunieron solas o combinadas el $26,6 \%$. Un $14,7 \%$ se ubica en "no aplica", dado que no corregían y un $6 \%$ otras respuestas, tal como se puede apreciar en el Gráfico 1.

Gráfico 1.- Emociones en la corrección

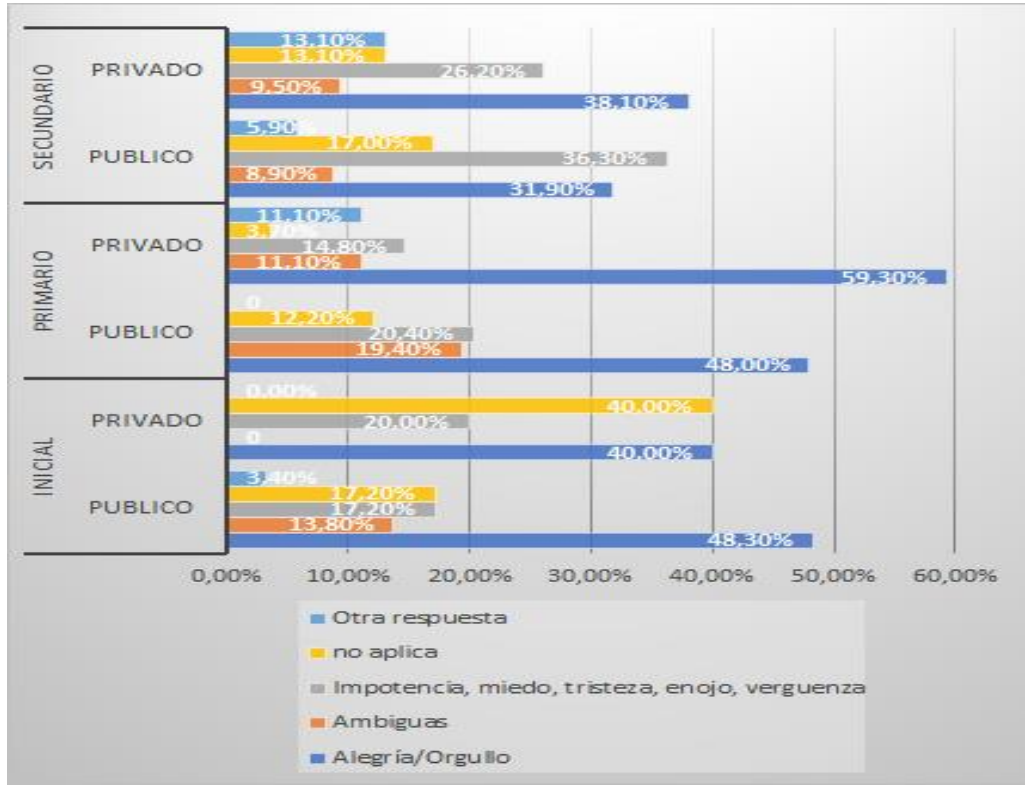

Fuente: elaboración propia.

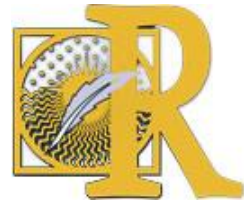

Fecha de recepción: 28-04-2021 Fecha de aceptación: 30-04-2021

Vergara, G., Fraire, V., Manavella, A. \& Salessi, S. Prácticas, percepciones y emociones de docentes de Argentina en tiempos de pandemia Covid-19

International Journal of Educational Research and Innovation (IJERI), 15, 568-584

ISSN: 2386-4303 DOI https://doi.org/10.46661/ijeri.5903 
El porcentaje más elevado de alegría y orgullo se concentra en el primario privado $(59,3 \%)$, seguido por inicial público $(48,3 \%)$, primario público (48\%) e inicial privado. El mayor porcentaje de emociones "ambiguas" está en el primario público, mientras que impotencia y miedo se destacan en el secundario público (36,3\%). El análisis de la prueba Chi Cuadrado de Pearson muestra que hay una asociación estadísticamente significativa entre emociones y tipo de gestión $\left(X^{2}=11.62 ; p<0.05\right)$, así como entre emociones y nivel educativo $\left(X^{2}=26\right.$. $92 ; \mathrm{p}<0.05)$.

\section{Discusión.}

Las prácticas de un trabajo fuertemente modificado en tiempos de ASPO implican continuidades y metamorfosis, en el marco de procesos de estructuración social. La incorporación de tecnología en la vida cotidiana y, paulatinamente, en la educación constituyen aspectos previos a la pandemia y, por lo tanto, presentes en el hacer diario de los y las docentes. Las referencias espacio/temporales inciden en la configuración de las sensibilidades sociales (Scribano, 2010), que se arman entre emociones y percepciones, anudadas en las prácticas.

Un conjunto de resultados obtenidos en este estudio, entran en diálogo con investigaciones previas. Tal el caso de los aportes de Gómez Dávalos y Rodríguez Fernández (2020) en cuanto a la sobrecarga de tareas. Los resultados aquí presentados muestran que casi la mitad $(46.3 \%)$ de la muestra trabajó más de 32 horas semanales. Pero además, aportó información sobre la distribución del tiempo, diferente y completamente diferente para el 83\% de los casos, lo cual se conecta con los estudios del home office y el trabajo digital (Scribano y Lisdero, 2019).

Prácticas de un trabajo que cambia abruptamente y se intensifica, se tensionan con percepciones de adecuación y emociones de alegría y orgullo, lo que podría ser una vía para explorar la presencia de mecanismos de soportabilidad social (Scribano, 2007), en un contexto de pérdida de puestos de trabajo pero sin ser "trabajo esencial"3.

Picón et al. (2020) mostraron que las tareas efectuadas con mayor frecuencia fueron la revisión y evaluación del desarrollo de contenidos. Los resultados obtenidos en la encuesta también dan cuenta de esto en el $64 \%$ de los casos, aportando sobre las modalidades de corrección. Lo motivacional/emocional (contención y acompañamiento a estudiantes) decrece a medida que se avanza en los niveles, junto con evaluar algunos aspectos importantes y nuevas habilidades sobre todo en el nivel secundario. Esto interroga acerca de "lo necesario" de la contención desde las instituciones educativas, en el marco de un proceso de gestión de las emociones que se daba previamente. Las diferencias entre niveles resultan esperables por las etapas de desarrollo cognitivo/afectivo de los y las estudiantes, no obstante ello, aparece como un componente transversal en la educación, pese a que casi el $40 \%$ en el secundario indicó corregir sólo contenido.

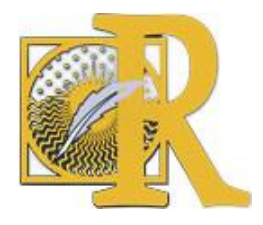

Fecha de recepción: 28-04-2021 Fecha de aceptación: 30-04-2021 
Una mirada positiva afirma que un desarrollo integral abarca las necesidades emocionales y sociales junto con el promover mayor conciencia de las emociones propias como una vía para favorecer un clima saludable en la clase (Bernal Guerrero y Cárdenas Gutiérrez, 2009; Cabello et al., 2010 y Hernández Barraza, 2017). Al mismo tiempo, la educación en ambientes virtuales, en especial los que promueven el trabajo colaborativo, contribuyen al fortalecimiento de estas habilidades socioemocionales mencionadas (Chiappe y Consuelo Cuesta, 2013).

En línea con Baptista Lucio et al. (2020) de que el 65\% tuvo sentimientos positivos o, satisfacción por lo que realizaban, en esta encuesta alegría y orgullo reflejaron las emociones en 4 de cada 10 docente, y se observaron mayores emociones negativas (impotencia, miedo, enojo, tristeza, vergüenza) en el secundario y más en el público. Emociones ambiguas presentaron mayor intensidad en el primario público.

Entre las principales limitaciones de este análisis hay que citar la representatividad de la muestra, que al haber sido seleccionada en forma no probabilística impide la generalización de los resultados a la población en su conjunto. En este sentido, la muestra bajo estudio quedó conformada por una mayoría de mujeres, entre 40-49 años. Dentro de este grupo, el $75 \%$ estaba casada/o, con 2 proveedores (73\%) y el 38\% tenía de 2 hijos. El $68 \%$ se desempeñaba en un establecimiento de gestión pública y, un $25 \%$ en gestión privada con aporte estatal. El $57 \%$ estaba en nivel secundario, el $33 \%$ en primario y el $10 \%$ en inicial. Cabe señalar que, según relevamientos de 2017, había 147.169 cargos en el nivel inicial, 365.761 en el primario y 204.186 en el secundario. En cuanto al género, según Censo Nacional del Personal de los Establecimientos Educativos (CENPE) de 2014, el 76,5\% son mujeres. La mitad tenía entre 35 y 49 años. (Buchbinder et al, 2019).

Asimismo, dado que los datos fueron recabados con un instrumento autoadministrado, las respuestas podrían verse contaminadas por la deseabilidad social como por otros componentes subjetivos. Para superar esta limitación sería valioso que futuros estudios evalúen la posibilidad de incluir datos provenientes de otras fuentes, con el propósito de incrementar la validez externa.

Más allá de sus limitaciones, cabe destacar que esta investigación constituye un aporte original, al analizar las prácticas de corrección desde la perspectiva de la sociología de los cuerpos y emociones, en relación con estudios sobre el trabajo y, la educación.

\section{Conclusiones.}

En este artículo se analizaron resultados de prácticas, percepciones y emociones en el corregir de docentes de tres provincias de Argentina en tiempos de ASPO por la pandemia Covid-19. La docencia en general y las instancias de corrección/evaluación implicaron cambios repentinos y abruptos.

Dichos cambios pueden ser entendidos desde una Sociología de los cuerpos y las emociones, como prácticas inscriptas en procesos previos que se modificaron espacio/temporalmente, implicando posiblemente tensiones en las percepciones y emociones.

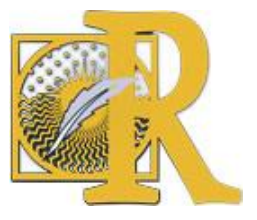

Fecha de recepción: 28-04-2021 Fecha de aceptación: 30-04-2021 Vergara, G., Fraire, V., Manavella, A. \& Salessi, S. Prácticas, percepciones y emociones de docentes de Argentina en tiempos de pandemia Covid-19 
Tal como se observa en los datos analizados por niveles educativos, se advierte una conexión entre prácticas de corrección que se incrementaron de la mano de emociones cercanas a la impotencia y tristeza en el nivel secundario. Nivel que mostró mayor carga de horas y mayor diferencia en la distribución horaria del trabajo. Y si bien se percibe las correcciones como adecuadas no se reportaron los mismos resultados a la hora de valorar si se cumple con la labor docente o se hace apenas lo que se puede. Esta última opción se conecta con la impotencia y alerta acerca de las dificultades en el proceso de enseñanza remota.

Es decir que, en general, los esquemas de clasificación y valoración dan cuenta de disposición a trabajar en circunstancias diferentes, de estar cumpliendo con sus tareas y de hacerlo de modo adecuado. Dichas percepciones se articulan con una práctica caracterizada por la intensificación y cambios entre menos contenidos, emociones y nuevas habilidades

En cuanto a las emociones, se advierte una relación entre los ejes de la tristeza y la impotencia, donde más carga de trabajo hay, aunque las autopercepciones eran positivas. Alegría y orgullo tienen más presencia en nivel inicial y primario, apareciendo mayor impotencia y enojo en el nivel secundario.

Se considera que las relaciones entre prácticas, percepciones y emociones permiten profundizar las experiencias del trabajo docente en tiempos de pandemia, atendiendo a las especificidades y complejidad de cada nivel educativo. Se requiere profundizar en relación con lo enseñado y los dispositivos tecnológicos a fin de aportar en aras de una experiencia que seguramente dejará huellas en la educación de las próximas décadas.

\section{Financiamiento.}

El presente artículo se inscribe en el marco del proyecto de investigación "Prácticas y percepciones del mundo del trabajo en el siglo XXI: continuidades, metamorfosis y 'nuevos trabajos' en Rafaela (2019-2021)" aprobado y financiado por la Universidad Nacional de Rafaela (Santa Fe, Argentina), para el período 2019-2021.

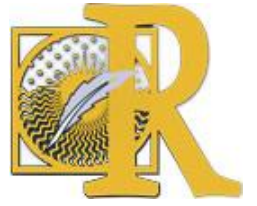

Fecha de recepción: 28-04-2021 Fecha de aceptación: 30-04-2021 
Notas.

${ }^{1}$ En Argentina, el Ministerio de Educación de la Nación y el Consejo Federal de Educación, implementaron el programa "Seguimos Educando", a partir del cual se procedió a la continuidad pedagógica nacional a través de un sistema multiplataforma. Mediante este programa, la navegación por la plataforma digital pasó a no consumir datos, al mismo tiempo que se aseguró la producción y distribución de materiales impresos para aquellas comunidades educativas que carecían de acceso a internet. También se emitieron 14 horas diarias de contenidos educativos televisivos y siete horas diarias de radio para todos los niveles educativos. Por su parte, cada provincia desarrolló diversas propuestas y estrategias a fin de asegurar la continuidad educativa de cada ciudadano (Observatorio de Políticas Públicas, 2020). Desde UNICEF se creó y distribuyó un manual titulado "los equipos de conducción frente al COVID-19: claves para acompañar y orientar a los docentes, las familias y los estudiantes en contextos de emergencia". En dicha serie se presentan desafíos y propuestas, vinculadas a la contención emocional de los diversos miembros que conforman la comunidad educativa Argentina (Kaplan, 2020).

2 Dado que los datos que se describen y analizan en el escrito se recogieron durante junio de 2020 en Argentina, se considera pertinente mencionar la situación epidemiológica del país en dicho mes. El día 30 de junio de 2020, según los datos oficiales aportados por el Ministerio de Salud de la Nación Argentina, se habían registrado un total de 64530 casos positivos de COVID-19 en el país. Del total de casos en el momento en el que se realizó el recorte, 1.065 $(1,7 \%)$ eran importados, $23565(36,1 \%)$ correspondían a contactos estrechos de casos confirmados, $28.732(44,5 \%)$ remitían a casos de circulación comunitaria y el resto se encontraban en investigación epidemiológica. Al momento, la cantidad de personas fallecidas en el país que presentaban COVID-19 eran 1.307. Hasta el 30 de junio, el número de casos acumulados en Buenos Aires era 31639, en Córdoba 648 y en Santa Fe 421.

${ }^{3}$ Según el Decreto presidencial 297/2020 del 19 de marzo de 2020. El texto del mismo se encuentra disponible en: https://www.boletinoficial.gob.ar/detalleAviso/primera/227042/20200320 . Último acceso, 30/04/2021.

\section{Referencias.}

- Antunes, R. (2005). Los sentidos del trabajo: ensayo sobre la afirmación y negación del trabajo. Buenos Aires: Herramienta Ediciones.

- Baptista Lucio, P.; Almazán Zimerman, A.; Loeza Altamirano, C. A.; López Alcaraz, V. A. y Cárdenas Domínguez, J. L. (2020). Encuesta Nacional a Docentes ante el COVID-19. Retos para la educación a distancia. Revista Latinoamericana De Estudios Educativos, 50 (ESPECIAL), 41-88. DOI: https://doi.org/10.48102/rlee.2020.50.ESPECIAL.96

- Bericat Alastuey, E. (2000). La sociología de la emoción y la emoción en la sociología. Papers. Revista de Sociología, 62, 145-176. DOI: http://dx.doi.org/10.5565/rev/papers/v62n0.1070

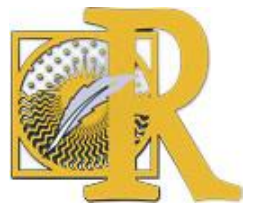

Fecha de recepción: 28-04-2021 Fecha de aceptación: 30-04-2021 
- Bernal Guerrero, A. y Cárdenas Gutiérrez, A. R. (2009). Influencia de la competencia emocional docente en la formación de procesos motivacionales e identitarios en estudiantes de Educación Secundaria. Una aproximación desde la memoria autobiográfica del alumnado. Revista de investigación educativa, 27(1), 203-222. Recuperado de: https://revistas.um.es/rie/article/view/94371

- Bourdieu, P. (1999). Meditaciones pascalianas. Barcelona: Anagrama.

- Bourdieu, P. (2003) Creencia Artística y bienes simbólicos. Elementos para una sociología de la cultura. Córdoba-Buenos Aires: Aurelia*Rivera.

- Buchbinder, N.; McCallum, A. y Volman, V. (2019). El estado de la educación en la Argentina. Observatorio Argentinos por la Educación. Recuperado de: https://cms.argentinosporlaeducacion.org/media/reports/El estado de la educacion Arg entina.pdf

- Cabello, R., Ruiz-Aranda, D. y Fernández-Berrocal, P. (2010). Docentes emocionalmente inteligentes. Revista Electrónica Interuniversitaria de Formación del Profesorado, 13 (1), 41-49. Recuperado de: https://www.redalyc.org/articulo.oa?id=2170/217014922005

- Chiappe, A. y Consuelo Cuesta, J. (2013). Fortalecimiento de las habilidades emocionales de los educadores: interacción en los ambientes virtuales. Educación y Educadores, 16(3), 503-524.Recuperado de: https://www.redalyc.org/articulo.oa?id=834/83429830006

- Decreto presidencial 297/2020. [Presidencia de la Nación Argentina en acuerdo general de Ministros]. Aislamiento Social, Preventivo y Obligatorio. 19 de marzo de 2020. Recuperado de: https://www.boletinoficial.gob.ar/detalleAviso/primera/227042/20200320

- Fraire, V. (2015). Resignación y orgullo. Una aproximación a las emociones en torno a lo barrial y su vinculación con procesos de estructuración social, en G. Magallanes, C. Gandía y G. Vergara (comps.). Expresiones/experiencias en tiempos de carnaval, pp. 177-206. Buenos Aires: CICCUS.

- Gómez Dávalos, N. R. y Rodríguez Fernández, P. (2020). Estrés en docentes en el contexto de la pandemia de COVID-19 y la educación. Academic Disclosure, 1 (1 special edition Covid-19), 216-234. Recuperado de: https://revistascientificas.una.py/ojs/index.php/rfenob/article/view/150/124

- Hernández Barraza, V. (2017). Las competencias emocionales del docente y su desempeño profesional. Alternativas en psicología, 37, 79-92. Recuperado de: https://www.alternativas.me/attachments/article/147/06\%20-

\%20Las\%20competencias\%20emocionales\%20del\%20docente.pdf

- Kaplan, C. V. (2020). Contención emocional de equipos directivos y herramientas para docentes y familia: el acompañamiento a la comunidad educativa en un contexto de emergencia. Buenos Aires: UNICEF. Recuperado de: https://www.unicef.org/argentina/media/8436/file/Serie-Conduccion-Covid-3.pdf

- Le Breton, D. (2002). Antropología del cuerpo y la modernidad. Buenos Aires: Nueva Visión.

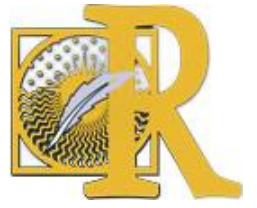

Fecha de recepción: 28-04-2021 Fecha de aceptación: 30-04-2021 Vergara, G., Fraire, V., Manavella, A. \& Salessi, S. Prácticas, percepciones y emociones de docentes de Argentina en tiempos de pandemia Covid-19 
- Luna Zamora, R. (2010). La Sociología de las emociones como campo disciplinario. Interacciones y estructuras sociales. En A. Scribano y P. Lisdero (comps.). Sensibilidades en juego: miradas múltiples desde los estudios de los cuerpos sociales y las emociones, pp.15-38. Córdoba: CEA-CONICET.

- Marx, K. (2004). Manuscritos Económico-Filosóficos de 1844. Buenos Aires: Colihue.

- Ministerio de Salud de la Nación Argentina (2020). Información epidemiológica. Recuperado de: https://www.argentina.gob.ar/salud/coronavirus-COVID-19/sala-situacion

- Narodowsky, M.; Volman, V. y Braga, F. (2020). Tiempo destinado a actividades escolares y acompañamiento adulto (tercer informe). En La educación argentina durante la pandemia de COVID-19, pp. 1-11. Observatorio Argentino por la educación. Recuperado de: https://cms.argentinosporlaeducacion.org/media/reports/Tiempo destinado a actividade s escolares y ayuda de adultos ArgxEdu.pdf

- Observatorio de Políticas Educativas (2020). La escolarización en tiempos de pandemia. Entre Ríos: Facultad de Ciencias de la Educación, UNER. Recuperado de: https://www.fcedu.uner.edu.ar/observatorio/wp-content/uploads/sites/6/2020/04/Art\%C3\%ADculo Laescolarizaci\%C3\%B3n-en-tiempos-de-pandemia.pdf

- Oducado, R. M.; Parreño-Lachica, G. y Rabacal, J. (2021). Estrés percibido debido a la pandemia de COVID-19 entre los profesores profesionales empleados. IJERI: Revista internacional de investigación e innovación educativas, (15), 305-316. DOI: https://doi.org/10.46661/ijeri.5284

- Pérez Pino, M.; Enrique Clavero, J. O.; Carbó Ayala, J. E. y González Falcón, M. (2017). La evaluación formativa en el proceso de enseñanza aprendizaje. EDUMECENTRO, 9 (3), 263-283. Recuperado de: http://scielo.sld.cu/scielo.php?script=sci arttext\&pid=S207728742017000300017\&lng=es\&tlng=es

- Picón, G. A.; González de Caballero, G. K. y Paredes Sánchez, J. N. (2020). Desempeño y formación docente en competencias digitales en clases no presenciales durante la pandemia COVID-19. Scientific Electronic Library Online, 1-16. DOI: https://doi. org/10.1590/SciELOPreprints.778

- Resolución N¹08. Anexo I y II. (2020). [Ministerio de Educación de la Nación]. Nuevo Régimen Académico. Argentina. 16 de marzo de 2020.

- Resolución N³63. Anexo I y II. (2020). [Consejo Federal de Educación de Argentina]. Asamblea del Consejo Federal de Educación. Argentina. 19 de mayo de 2020.

- Scribano, A. (2007). La sociedad hecha callo: conflictividad, dolor social y regulación de las sensaciones. En A. Scribano (comp.). Mapeando interiores, pp. 119-143. Córdoba: Universitas.

- Scribano, A. (2010). Las sensibilidades prohibidas: el epílogo de un libro sobre la transformación social. En A. Scribano y P. Lisdero (comps.). Sensibilidades en juego: miradas múltiples desde los estudios sociales de los cuerpos y las emociones, pp. 246275. Córdoba: CEA-CONICET.

- Scribano, A. (2012). Sociología de los cuerpos/emociones. RELACES,10, 93-113. Recuperado de: https://dialnet.unirioja.es/servlet/articulo?codigo=6981013

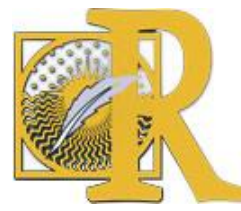

Fecha de recepción: 28-04-2021 Fecha de aceptación: 30-04-2021 Vergara, G., Fraire, V., Manavella, A. \& Salessi, S. Prácticas, percepciones y emociones de docentes de Argentina en tiempos de pandemia Covid-19 
- Scribano, A. y Lisdero, P. (edits). (2019). Digital Labour, Society and Politics of sensibilities. London: Palgrave McMillan. DOI: https://doi.org/10.1007/978-3-030-12306-2

- SITEAL (2020). Sistematización de respuestas de los sistemas educativos de América Latina a la crisis de la COVID-19. Buenos Aires: IIPE UNESCO. Recuperado de: https://www.siteal.iiep.unesco.org/respuestas educativas covid 19

- Turner, B. S. (1994). Los avances recientes en la teoría del cuerpo. REIS: Revista Española de Investigaciones Sociológicas, 68, 11-40. Recuperado de: http://ih-vmcisreis.c.mad.interhost.com/REIS/PDF/REIS 068 04.pdf

- Vergara, G. (2011). Tramas corporales, percepciones y emociones en las mujeres recuperadoras de residuos de Córdoba (Argentina). En J. Ferreira y A. Scribano (edits.). Corpos em concerto: diferenças, desigualdades y desconformidades, pp. 273-318. Recife: Editora Universitária UFPE.

- Vergara, G. (2013). Reflexiones sobre las contribuciones de la observación participante para una Sociología de los cuerpos y las emociones. Revista Latinoamericana de Metodología de la Investigación Social, 6, 42-56. Recuperado de: http://relmis.com.ar/ojs/index.php/relmis/article/view/92/95

- Vergara. G. (2015). Mujeres recuperadoras de residuos entre familias y trabajo: la percepción de proveer como amas de casa (Córdoba, 2006-2013). En G. Vergara (comp.). Recuperadores, residuos y mediaciones. Análisis desde los interiores de la cotidianeidad, la gestión y la estructuración social, pp.229-259. CABA: ESE Editora. 